
\title{
$\begin{array}{ll}\text { Research Square } & \begin{array}{l}\text { Preprints are preliminary reports that have not undergone peer review. } \\ \text { They should not be considered conclusive, used to inform clinical practice, } \\ \text { or referenced by the media as validated information. }\end{array}\end{array}$
}

\section{Human multipotent mesenchymal stromal cell transcriptome changes in response to PDGF-BB}

\section{Olga Grigoreva}

Institute for Regenerative Medicine, Medical Research and Education Center, Lomonosov Moscow State

University

\section{Mikhail Arbatsky}

Faculty of Medicine, Lomonosov Moscow State University

\section{Ekaterina Novoseletskaya}

Faculty of Medicine, Lomonosov Moscow State University

\section{Alexander Ishkin}

Discovery Science, Clarivate Analytics

\section{Natalia Kalinina}

Faculty of Medicine, Lomonosov Moscow State University

\section{Anastasia Efimenko ( $\nabla$ efimenkoan@gmail.com )}

Institute for Regenerative Medicine, Medical Research and Education Center, Lomonosov Moscow State University

\section{Research Article}

Keywords: multipotent mesenchymal stromal cells (MSCs), Platelet-derived growth factor (PDGF-BB), tissue damage, sequence markers

Posted Date: May 5th, 2021

DOI: https://doi.org/10.21203/rs.3.rs-413033/v1

License: (c) (i) This work is licensed under a Creative Commons Attribution 4.0 International License. Read Full License 


\section{Abstract}

Activation of multipotent mesenchymal stromal cells (MSCs) is a central part of tissue response to the damage. Platelet-derived growth factor (PDGF-BB), which is abundantly released in the damaged area, potently stimulates the proliferation and migration of MSCs. Recent evidences indicate that tissue injury associates with the accumulation of senescent cells, including ones of MSC origin. Therefore, we hypothesized that PDGF-BB induces MSC senescence. To evaluate mechanisms of early activation of MSCs by PDGF-BB, we performed transcriptome profiling of human MSCs isolated from adipose tissue after exposure to PDGF-BB for 12 and 24 hours. We demonstrated that PDGF-BB induced the expression of senescence markers in MSCs. However, further experimental validation of transcriptomic data clearly indicated that PDGF-BB exerts mitogenic and pro-migratory effects on MSCs, but did not significantly stimulate their senescence.

\section{Introduction}

Recently, tissue injury was associated with the accumulation of senescent cells, including those originating from multipotent mesenchymal stem/stromal cells (MSCs) (Neri and Borzì, 2020). Numerous studies have implicated MSCs to the tissue regeneration upon damage, with paracrine activity being a central mechanism of their action (Pittenger, 2019; Sagaradze, 2020; Basalova, 2020). Signals from damaged tissues could both activate MSCs and cause the acquisition of a senescent phenotype characterized by cell

cycle arrest with p16 ${ }^{\text {INK4a }}$, p21 Waf1/Cip1 and p53 expression activation, resistance to apoptosis, and specific senescence-associated secretory phenotype (SASP) activated by transcription factors NF-KB and C/EBP $\beta$ (Coppe, 2010; Campisi, 2019; Fafián-Labora, 2020). SASP is characterized by a shift towards a proinflammatory, mitogenic cytokines as well as changes in the extracellular matrix composition. MSC senescence caused the crucial impairment of their regenerative capacity and regulatory functions.

Upon a damage MSCs respond to a multitude of factors, including abundantly presented platelet-derived growth factor (PDGF). These cells express PDGFRß receptor, which has the highest affinity for PDGF-BB dimer (Heidaran, 1991). PDGF can activate both migration and proliferation of stromal cells (De Donatis, 2008), therefore stimulating tissue regeneration. However, PDGF-mediated activation of stromal cells could also contribute to fibrogenesis (Klinkhammer, 2018).

Since proliferation and senescence are closely related, we hypothesized that PDGF-BB could simultaneously cause MSCs senescence. To test this hypothesis, we performed transcriptomic profiling of human adiposederived MSCs after treatment with PDGF-BB by RNA sequencing. Acquisition of senescent phenotype of MSCs treated by PDGF-BB was evaluated by beta-galactosidase and p21 expression as well as secretion of SASP components (IL-6, MCP-1, PAI-1). We confirmed mitogenic and pro-migratory effects of PDGF-BB on MSCs but did not observe the significant stimulation of MSC senescence in these conditions.

\section{Materials And Methods}


Human adipose-derived MSCs were isolated from subcutaneous adipose tissue obtained from 4 healthy donors during abdominal surgery. All donors or, if donors were under 18, a parent and/or legal guardian gave their informed consent and the local ethics committee of the Medical Research and Education Center of Lomonosov Moscow State University (Moscow, Russia) approved the study protocol (\#4, 04.06.2018). All methods were performed in accordance with the relevant guidelines and regulations.

Subcutaneous adipose tissue samples $(0,5-5 \mathrm{ml})$ harvested during surgery were homogenized and digested in collagenase I $(200 \mathrm{U} / \mathrm{ml}$, Worthington Biochemical) and dispase $(40 \mathrm{U} / \mathrm{ml}$, Sigma) solution under agitation for $30-40 \mathrm{~min}$ at $37^{\circ} \mathrm{C}$. Then tissue was centrifuged at $200 \mathrm{~g}$ for $10 \mathrm{~min}$ and the supernatant discarded. The pellet containing ADSC was lysed to destroy erythrocytes, filtered through a sieve (BD Falcon Cell Strainer, $100 \mathrm{mkm}$ ) and centrifuged at $200 \mathrm{~g}$ for $10 \mathrm{~min}$. The final pellet was resuspended in culture medium. Cells were cultured in AdvanceSTEM ${ }^{\mathrm{TM}}$ Mesenchymal Stem Cell Media containing $10 \%$ AdvanceSTEM $^{\text {Tm }}$ Supplement (HyClone, USA), $1 \%$ antibiotic-antimycotic solution (HyClone) at $37{ }^{\circ} \mathrm{C}$ in $5 \%$ $\mathrm{CO} 2$ incubator. Cells were passaged at $70 \%$ confluency using HyQTase solution (HyClone). For the experiments, MSCs of the third or fourth passages were seeded at a density of $7 \times 10^{\wedge} 3$ cells $/ \mathrm{cm} 2$ on uncoated culture plates (Corning, USA) a day before the experiment. At these passages phenotypic characterization and functional evaluation of mesenchymal properties were performed as described below. MSCs were washed thoroughly with HBSS (HyClone, USA) to remove any residues of AdvanceSTEM ${ }^{\text {TM }}$ Supplement. Then HBSS was replaced by serum-free AdvanceSTEM ${ }^{\text {TM }}$ Mesenchymal Stem Cell Media and MSCs were cultivated overnight. In selected experiments $10 \mathrm{ng} / \mathrm{ml}$ PDGF-BB were added 18 hours after deprivation. For assessment of transcriptome content MSCs were cultured under standard conditions (5\% $\mathrm{CO} 2$ and $21 \% \mathrm{02}$ ) at $37^{\circ} \mathrm{C}$ for 12 or $24 \mathrm{~h}$ with or without PDGF-BB. At the end of the incubation cells were washed with HBSS and were lysed in the RLT buffer for further isolation of the total RNA. MSCs quantity and viability were assessed at the end of experiment using Countess cell counter (Invitrogen).

\section{MSC immunophenotyping}

MSC immunophenotype was analysed using flow cytometry. After medium harvesting cells were detached from culture dishes using EDTA solution and stained with antibodies against CD14 (eBioscience, № 140141-81), CD34 (BD Pharmingen, № 555824), CD45 (BD Pharmingen, 340953), CD73 (BD Pharmingen, № 550257), CD90 (BD Pharmingen, № 555597) and CD105 (BD Pharmingen, № 560819) as described elsewhere. IgGs of appropriate isotype were used as a negative control. Stained cells were analyzed using MoFlo cell sorter and Summit software (Dako Cytomation, Denmark).

\section{MSC differentiation assays}

MSC abilities to differentiate into osteogenic, adipogenic and chondrogenic directions were tested in vitro using standard differentiation and analysis protocols described elsewhere. Briefly, osteogenic differentiation was induced by plating $6 \times 10^{\wedge} 4$ of MSCs into 24-well plate and incubating in growth medium containing 10^-8 M dexametasone, $10 \mathrm{mM}$ ß-glycerol-2-phosphate, $0.2 \mathrm{mM}$ 2-phospho-L-ascorbic acid (Invitrogen, USA) during 3 weeks. Differentiation efficiency was analyzed using Alizarin Red S staining. Adipogenic differentiation was induced by 3 cycles of consecutive incubation for 6 days in the growth medium

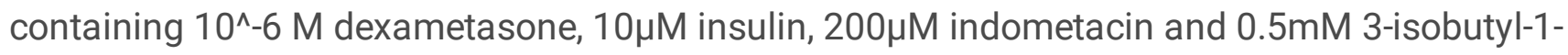


methylxantine (Invitrogen, USA) followed by 3 days incubation in growth medium containing $10 \mu \mathrm{M}$ insulin. Accumulation of intracellular lipids was assessed by Oil-Red-O staining. Chondrogenic differentiation was induced by incubation of pelleted MSCs in AdvanceSTEM Chondrogenic Differentiation Medium (HyClone) for 28 days and stained with toluidine blue.

\section{HUVEC isolation and culture}

Human umbilical cord endothelial cells (HUVEC) were obtained from cell culture Depository of Live Systems (Lomonosov Moscow State University), ID of the collection MSU_HUVEC. HUVEC were cultured in EGM ${ }^{\text {TM }}$ Media containing $2 \%$ fetal bovine serum (FBS) and recombinant growth factors (CC-4176, Lonza, Switzerland), at $37^{\circ} \mathrm{C}$ in $5 \% \mathrm{CO} 2$ incubator. Cells were passaged at $70 \%$ confluency using HyQTase solution (HyClone). For migration assay cells were used between passage 3-6.

\section{RNA isolation and transcriptome analysis}

Total cellular RNA was isolated from cultured MSCs using RNeasy Kit (Qiagen, cat \# 74104) according to manufacturer instructions. Five hundred nanograms of total RNA was used for transcriptome analysis.

\section{Whole-Genome Expression Analysis Using the Sentrix® Human-6 Expression BeadChips}

Whole-genome gene expression was assessed using a direct hybridization approach. To amplify starting material, a single-round IVT amplification was carried out using the Illumina RNA Amplification Kit, manufactured by Ambion ${ }^{\circledR}$, Inc.

The HumanWG-6 BeadChip targets, NM Coding transcript, well-established annotation 27,455, XM Coding transcript, provisional annotation 7,870, NR Non-coding transcript, well-established annotation.

446, XR Non-coding transcript, provisional annotation 196, includes 12,837 probes targeting EST clusters from the UniGene database (Build 199). As a result, the HumanWG-6 BeadChip targets a total of 25,440 annotated genes with more than 48,000 probes (Whole-Genome Expression Analysis Using the Sentrix ${ }^{\circledR}$ Human- 6 and HumanRef-8 Expression BeadChips)

(https://www.illumina.com/documents/products/techbulletins/techbulletin_whole_genome_expression.pdf)

\section{Data preprocessing}

Illumina BeadStudio output file was processed using lumi package available in Bioconductor software collection. The processing included following steps: Quality control of raw data; Background subtraction; Variance stabilization using algorithm VST, developed for Illumina data. Values after this processing step should be treated as log-transformed. Quantile normalization.

First, pairwise correlations of intensities were consistently high (>0.95) for the samples.

$p-$ value $=\frac{R ! n !(N-R) !(N-n) !}{N !} \sum_{i=\max (,, R+n-N)}^{\min (n, R)} \frac{1}{i !(R-i) !(n-i) !(N-R-n+i) i}$

Additionally, we excluded all probes that had detection p-value $>0.05$ in all 3 samples. 
4 columns (samples) and 11073 rows (probes) corresponding to 9659 non-redundant gene.

\section{Fold change calculation}

Calculated fold changes of gene expression relative to the expression in control sample.

\section{Enrichment analysis}

Enrichment analysis was conducted with the GOstats package (4) of Bioconductor. This analysis aims to identify biological processes (in our case, "BP" branch of Gene Ontology (5) biological processes tree) which are significantly associated with observed expression changes.

The significance of enrichment is defined by p-values of hypergeometric distribution:

$p-$ value $=\frac{R ! n !(N-R) !(N-n) !}{N !} \sum_{i=\max (r, R+n-N)}^{\min (n, R)} \frac{1}{i !(R-i) !(n-i) !(N-R-n+i) i}$

where,

$\mathrm{N}$ - number of genes in the whole data set

$\mathrm{R}$ - number of genes in a gene list of interest

$\mathrm{n}$ - number of genes associated with a particular process from the ontology

$r$ - number of gene from input list intersection with genes from a particular category

\section{Data Records}

CHIP files associated with the samples analysed in this study are deposited at gene expression omnibus with the accession number GSE152578.

\section{Immunohistochemistry}

Human adipose-derived MSCs from 3 healthy donors were seeded at density $10^{\wedge} 4$ per $\mathrm{ml}$. Cell were used between passage 3 and 4. PDGF BB were added at concentration $10 \mathrm{ng} / \mathrm{ml}$ for 24 hours after 18 hours of serum deprivation. MSCs were fixed with $4 \%$ paraformaldehyde solution (Panreac, Spain) at room temperature for 10 min and incubated with 0,2\% triton X100 (Sigma, USA) solution at RT for 10 min. Further, MSCs were incubated for 1 hour in 1\% bovine serum albumin (BSA, Sigma, USA), 10\% normal goat serum (Abcam, UK) solution at room temperature to block the non-specific interaction of antibodies. Subsequently, the samples were incubated with primary polyclonal rabbit antibody for p21 Waf1/Cip1 (Cell Signalling, $2947 S$ ) or rabbit polyclonal IgG (Biolegend, 910801$)$ in $1 \%$ BSA solution at $+4^{\circ}$ overnight. Then samples were incubated with fluorescence labeled goat-anti-rabbit secondary antibodies (A11034, Invitrogen) at room temperature for $1 \mathrm{~h}$. Cell nuclei were labeled with DAPI (DAKO). Samples were analyzed with a Leica 
DM6000B fluorescent microscope equipped with a Leica DFC 360FX camera (Leica Microsystems GmbH, Germany). The percent of p21-positive MSCs was evaluated.

\section{$\beta$-galactosidase staining}

Evaluation of $\beta$-galactosidase activity was proceed with the Senescence $\beta$-Galactosidase Staining Kit (Cell Signalling, 9860) according to manufacturer's recommendations.

\section{ELISA assay}

MSCs from 3 healthy donors were used between passages 3-4. Cells were seeded at density $10^{\wedge} 4$ per ml and allowed to attach to plastic. After 18 hours of serum deprivation PDGF BB were added at concentration $10 \mathrm{ng} / \mathrm{ml}$ for 24 hours. MSCs were washed thoroughly with HBSS (HyClone, USA), then HBSS was replaced with DMEM low glucose (Gibco, USA) with $1 \%$ BSA and MSCs were cultivated for 72 hours. Condition medium was collected and centrifuged at $300 \mathrm{~g}$ for $10 \mathrm{~min}$ to remove cellular debris. Supernatant was transferred to clear tube with with $1 \%$ protease inhibitor cocktail x100 (Sigma Aldrich). The concentration of IL-6, MCP-1 (CCL-2), PAl-1, HGF, VEGF in the samples of conditioned medium was evaluated with ELISA kit (R\&D Systems) according to manufacturer's recommendations and normalized to cell number.

\section{Migration assay}

HUVEC migration assay was performed in automated RTCA xCELLigence ${ }^{T M}$ system (ACEA Bioscienses, USA) according to manufacturer's recommendations. Briefly, HUVEC media was changed to basal media EBM (Lonza, Switzerland) with 0,5\% bovine serum albumin (BSA, Imtek, Russia) to harvest serum growth factors 4-6 hours before experiment. The lower chamber of CIM-plate was filled with EBM with 0,5\% BSA as a negative control, EGM2, containing $2 \%$ of fetal bovine serum (FBS) (Lonza, Switzerland), conditioned media from MSC (CM MSC) or conditioned media from MSC pretreated with $10 \mathrm{ng} / \mathrm{ml} \mathrm{PDGF} \mathrm{BB} \mathrm{for} 24$ hours. As a control of possible chemotactic activity of PDGF itself it was added to one of the chambers in concentration $10 \mathrm{ng} / \mathrm{ml}$ in EBM 0,5\% BSA. 20000 of HUVEC were placed in the upper chamber. After placing the CIM plate into RTCA xCELLigence system bioimpedance on each well was fixed every 15 minutes. Data were processed with RTCA Software Pro (ACEA Biosciences, USA).

\section{Statistical analysis}

We have performed at least three independent experiments for each measurement. Statistical data processing was performed using the program GraphPad Prizm 8.0. Means \pm standard deviation (SD) were used to present quantitative data. The comparison of independent groups was performed by MannWhitney test. Differences were considered statistically significant at the significance level of $p<0.05$.

\section{Results}

PDGF-BB up-regulates genes associated with proliferation and cell cycle and down-regulates immune response-related pathways in MSCs 
MSCs isolated from the adipose tissue of healthy donors comply with recommendations of the International Society for Cellular Therapy (Dominici et al., 2006). These cells adhere to the culture plastic,

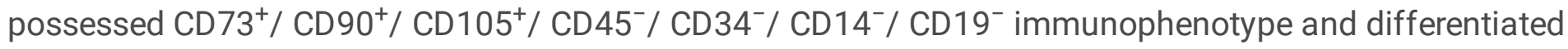
into osteogenic, chondrogenic and adipogenic lineages upon culturing in the induction medium (Fig. 1). Most cells were also positive for PDGFR $\beta$ (87-95\%).

1. Evaluation of MSC markers by flow cytometry. More than $99 \%$ of MSCs express CD73, CD90, CD105 on cell surface.

2. MSCs differentiate into osteocytes (Alizarin Red S staining), adipocytes (Oil-Red-O staining) and chondrocytes (Toluidine Blue staining) under inductive conditions. Representative images, phase contrast microscopy.

To evaluate the effect of PDGF-BB on global changes of gene expression in MSCs, we performed expression profiling of cells treated by PDGF for 12 of 24 hours using RNA sequencing. Differential expression analysis revealed numerous expression changes induced by PDGF treatment (152 and 127 genes were upregulated at least 2-fold after 12- and 24-hour exposure to PDGF-BB, correspondingly; 173 and 163 genes were downregulated at least 2-fold after 12- and 24-hour exposure to PDGF-BB).

Functional enrichment analysis was performed using clusterProfiler Bioconductor package. As a result, each differentially expressed gene list was associated with quantitatively ranked lists of Gene Ontology biological processes and KEGG pathways summarizing its effects at a systems level.

The top associated processes for a gene lists generated by FC $=2$ cutoff are presented at Fig. 2, 3 . Upregulated gene lists for experimental samples are clearly associated with proliferation and cell cycle. Among the genes of these groups, the expression was upregulated more than three times for CCNA2 (cyclin A2), CCNB2 (cyclin B2), CDC20 (cell division cycle 20), CDCA5 (cell division cycle associated 5), KIAA0101 (PCNA-associated factor), TOP2A (topoisomerase (DNA) II alpha), TYMS (thymidylate synthetase), AURKA (aurora kinase A), AURKB (aurora kinase B), NUSAP1 (nucleolar associated protein 1). Expression of these genes indicates that cells actively proliferate after PDGF treatment. This correlates with decreased

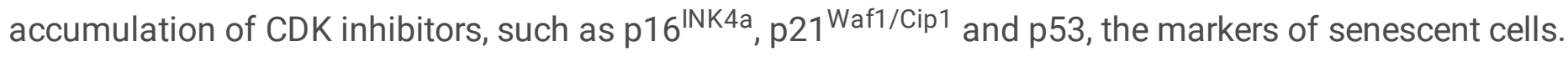
Additionally, we found significant increase in the expression of genes regulating MSC migration, such as PLAU (urokinase-type plasminogen activator, urokinase), PLAUR (urokinase-type plasminogen activator receptor, UPAR), MMP-1 (matrix metalloprotase-1) and SERPINE1 (plasminogen activator inhibitor-1, PAI-1), which were also related to SASP.

Downregulated genes were associated with immune response-related pathways and processes, most prominently gamma-interferon response. Significant decrease in expression was observed for chemokine genes, including CCL8 (C-C motif chemokine ligand 8, IL-8), CXCL10 (C-X-C motif chemokine ligand 10), CCL2 (C-C motif chemokine ligand, MCP-1), CXCL12 (C-X-C motif chemokine ligand). In addition, there was a strong decrease in IL6 gene expression, a high level of secretion of which is a characteristic feature of SASP. Among genes downregulated in MSCs after PDGF-BB treatment we also found THBS2 
(thrombospondine-2). Figure 3 illustrates similarities in enriched biological processes between 12- and 24hour treatments.

\section{PDGF-BB-treated MSCs did not acquire senescence biomarkers}

Senescent cells are characterized by accumulation of cell cycle inhibitors, such as p16 ${ }^{\text {INK4a }}$, p2 $1^{\text {Waf1/Cip1 }}$ and p53. We evaluated the number of MSCs with p21-positive nuclei with and without PDGF-BB treatment for 24 hours. We did not find significant differences between treated vs non-treated MSCs (Fig. 4, A). Furthermore, we evaluated the accumulation of senescent cells by activity of $\beta$-galactosidase. There was no significant elevation of $\beta$-gal activity in PDGF-BB treated MSCs (Fig. 4, B), which indicated that PDFG-BB did not induce cellular senescence.

Senescent associated secretory phenotype (SASP) is characterized by the elevated production of cytokines and interleukins, which mediates proinflammatory microenvironment of senescent cells. Among possible SASP factors in conditioned medium of MSCs we measured such crucial factors as IL-6, MCP-1 (CCL2) and PAI-1 by ELISA. Obtained protein concentrations were normalized by cell number. In case of IL-6 we found significant decrease in conditioned medium of PDGF-BB treated MSCs comparing to non-treated control. Concerning MCP-1 and PAl-1 there was no significant changes in levels of secreted proteins between treated vs non-treated cells (Fig. 4, C).

1. Immunocytochemical evaluation of p21 Waf1/Cip1 in control (CTRL) MSCs and after incubation with PDGF-BB for 24 hours. Fluorescent microscopy, green staining - p2 $1^{\text {Waf1/Cip1 }}$, blue staining - nuclei stained with DAPI. The percentage of p21 + cells was not changed significantly after PDGF-BB treatment.

2. Evaluation of $\beta$-galactosidase activity (blue staining) in control (CTRL) MSCs and after incubation with PDGF-BB. Phase contrast microscopy. The percentage of $\beta$-gal + cells was not changed significantly after PDGF-BB treatment.

3. MSC secretion of IL-6 decreased, while secretion of MCP-1 and PAI-1 was not changed significantly between control (CTRL) and after PDGF-BB treatment.

Data are presented as mean value of three independent experiments, error bars - standard deviation SD (B) or standart error SE (A, C), n.s. - no significant difference. Scale bar $100 \mu \mathrm{m}$.

\section{PDGF-BB stimulated MSC pro-angiogenic activity}

Angiogenic properties of MSCs mediated by cell paracrine factors are well described and have been shown to decrease with aging. We evaluated whether pro-angiogenic activity of MSC conditioned media is changed after PDGF-BB treatment using migration endothelial cells (HUVEC) test. HUVEC migrated in xCELLigence system towards basal media without additives as a negative control, EGM2 containing $2 \%$ of FBS as a positive control, conditioned media (CM) from MSCs or MSCs pretreated with PDGF-BB for 24 hours. We observed an increase in HUVEC migration towards CM from MSCs comparable with positive control (Fig. 5, A). Moreover, CM from MSCs pretreated with PDGF-BB stimulated the velocity of endothelial cells even stronger, while PDGF-BB itself decreased it (data not shown). The evaluation of the key pro-angiogenic 
growth factors, hepatocyte growth factor (HGF) and vascular endothelial growth factor (VEGF), demonstrated a significant increase in concentration of these factors in CM from PDGF-BB-treated MSCs comparing to CM from control MSCs (Fig. 5, B).

\section{Discussion}

PDGF-BB is a well-known mitogen for stromal cells, such as fibroblasts and MSCs. Produced in high concentrations during the acute phase of tissue damage, it activates stromal cell proliferation and migration (De Donatis, 2008). PDGFR $\beta$ receptor, which has the highest affinity for PDGF-BB dimer (Heidaran, 1991), is detected on many stromal cells, including MSCs. PDGF is an essential mitogen for many types of stromal cells involved in wound healing as it allows the cell to skip the $\mathrm{G} 1$ check point in the cell cycle (Song, 2007). During regeneration, PDGF attracts fibroblasts, monocytes, and macrophages into the damage zone, stimulates their proliferation and deposition of the temporary extracellular matrix, and then collagen I type, which forms the basis of the scar (Pierce, 1991). This process is essential for healing. However, apparently PDGF can trigger the secretion of endogenous factor by macrophages (and possibly other cells), which leads to the formation of an autocrine stimulation loop. Thus, both PDGF-mediated activation of the migration and proliferation of stromal cells into the lesion zone and the switching of the secretory cell phenotype can contribute to fibrogenesis and associated senescence (Klinkhammer, 2018).

Since aggressive surrounding in damaged zone may lead to induction of cellular senescence, we evaluated whether PDGF-BB could up-regulate senescence-related genes within human MSC transcriptional profile. Groups of up-regulated genes were mainly associated with activation of the cell cycle, stimulation of DNA replication, and cell division indicating that MSCs were actively proliferating after PDGF-BB treatment. It correlated with decreased accumulation of CDK inhibitors, such as p16INK4a, p21Waf1/Cip1 and p53, the markers of senescent cells. Indeed, in validation tests we have not found any significant elevation in the amount of p21-positive MSCs after PDGF-BB treatment. Many of the proteins coding by the up-regulated genes also modulate the reparative response of cells to DNA damage. Such results correlate with the general position on the role of PDGF-BB in the initial stages of response to damage as a crucial mitogen for MSCs.

Importantly, we observed significant increase in the expression of genes regulating MSC migration such as PLAU and its receptor PLAUR, MMP-1 and SERPINE1 (PAI-1). These genes are also found to be associated with increased cellular senescence. As it was described previously, accelerated aging is associated with cellular senescence and accompanied by marked increases in PAl-1 expression in tissues (Vaughan et al., 2017). PAI-1 is considered as a crucial component of SASP (Coppe et al., 2010). However, we did not observe any elevation of PAI-1 secretion by PDGF-BB treated MSCs at 24 hours point. uPAR as a cell-surface protein is broadly induced during senescence, and earlier we have demonstrated that its surface expression is increasing in MSCs isolated from adipose tissue of aged patients (Efimenko et al., 2014). Recently it was shown that uPAR-specific CAR-T cells efficiently ablate senescent cells in vitro and in vivo (Amor et al., 2020). On the other hand, both increased levels of UPA-UPAR axis and its inhibitor PAl-1 paradoxically can stimulate cell migration, probably through activation of different intracellular signalling (Pavon et al., 2016). 
Nevertheless, the increased secretion of PAI-1 by PDGF-BB-treated MSCs was not confirmed by ELISA in our validation tests.

Additionally, an increase in the expression of tubulin alpha and beta chain isoform genes stimulated by PDGF-BB treatment of MSCs indicates both the activation of proliferation and the possible rearrangement of the cytoskeleton during migration. Also, an increase in the expression of the HS3ST3A1 gene (heparin sulfate-glucosamine 3-sulfotransferase), an enzyme involved in the synthesis of heparan sulfate, which plays the role of an adapter in the stimulation of haptotaxis of MSCs through the activation of tyrosine kinase receptors, was detected (Veevers-Lowe et al., 2011). These data indicate the migration response of MSCs on the first day of PDGF-BB stimulation and the formation of the supporting mechanisms for homing stimulation.

Among genes down-regulated in human MSCs after PDGF-BB treatment we found THBS2 (thrombospondine-2), which was defined as a protein secreted by senescent cells. It inhibits endothelial migration and prevents cells from senescence escape (Guillon et al., 2019). However, we received data indicating that conditioned medium from MSC after PDGF-BB treatment had stimulatory effect on HUVEC directional migration comparing to initial MSC secretome. This may be due to the up-regulation of proangiogenic growth factor secretion by MSCs as well as down-regulation of THBS2 expression.

Analysis of clusters of down-regulated genes with 2-fold changes in expression in PDGF-BB-treated human MSCs showed a decrease in the activity of transcription of pro-inflammatory factors involved in the response to interferons, the innate immune response, and the response to viruses. In addition, there was a strong decrease in IL6 gene expression, a high level of secretion of which is a characteristic feature of SASP. These effects for IL6 and MCP-1 were confirmed by ELISA in the experimental validation tests. Obtained data support the acquisition of anti-inflammatory phenotype for MSCs under PDGF-BB, which is consistent with the idea of the formation of the immunosuppressive MSC phenotype under acute damage. According to the evaluation of widely recognized senescence biomarkers (p21 + and $\beta-g a l+)$ in MSCs we did not observe any significant difference between control cells and cells after PDGF-BB treatment. ELISA assay showed no significant increase in the level of SASP components, including IL-6, MCP-1 or PAI-1 after MSCs incubation with PDGF-BB.

It is important to note that cellular aging is a controversial process, and the development of senescent phenotype could have some benefits. In a young organism, after damage senescence of myofibroblasts promote the inhibition of their proliferation after healing, as well as the involvement of immune cells that eliminate myofibroblast. The critical contribution of senescent cells in wound healing, injury repair and tissue regeneration was demonstrated in several studies and related to so-called early senescence (Ritschka 2017; Campisi 2019). However, if the immune system does not cope with the elimination of senescent cells, they can accumulate in tissues promoting chronic pro-inflammatory microenvironment, fibrosis, or malignancy of adjacent healthy tissues (late senescence). Switching the normal MSC phenotype to senescent one can be determined by the entry of cells into the hostile environment after tissue damage, and PDGF-BB could play a critical role in this process. Summarizing our data, at short periods of exposure to PDGF-BB (up to 24 hours), the proliferation of MSCs is activated, the expression of genes associated with

Page 10/16 
migration increases, the level of secretion of growth factors increases, due to which MSCs paracrine stimulate endothelial migration and angiogenesis. However, analysis of senescent biomarkers in MSCs in such conditions indicate in favour the acquisition of early senescent phenotype, with benefits for tissue regeneration, but not the signs of late senescence.

\section{Declarations}

\section{Acknowledgements}

The study was supported by the Russian Foundation for Basic Research (grant \#19-29-04172).

\section{Author contributions}

Conceptualization and experimental design: G.O., K.N., E.A.; experimental data: G.O., N.E., E.A.; data analysis: G.O., A.M., I.A., K.N., E.A.; validation: G.O., N.E., E.A.; writing and editing: G.O., A.M., I.A., K.N., E.A. All authors have read and agreed to the published version of the manuscript.

\section{Competing interests}

The authors declare no competing financial interests.

\section{References}

1. Amor C., Feucht J., Leibold J. et al. Senolytic CAR T cells reverse senescence-associated pathologies. Nature (2020), in press.

2. Basalova N, Sagaradze G, Arbatskiy M, et al. Secretome of Mesenchymal Stromal Cells Prevents Myofibroblasts Differentiation by Transferring Fibrosis-Associated microRNAs within Extracellular Vesicles. Cells.

3. Campisi, J., Kapahi, P., Lithgow, G. J., Melov, S., Newman, J. C., and Verdin, E. From discoveries in ageing research to therapeutics for healthy ageing. Nature. 2019, 571,183-192.

4. Consortium GO. The Gene Ontology (GO) project in 2006. Nucl. Acids Res. 2006; 34(suppl_1): D322326.

5. Coppé JP, Desprez PY, Krtolica A, Campisi J. The senescence-associated secretory phenotype: the dark side of tumor suppression. Annu Rev Pathol. 2010;5:99-118.

6. De Donatis A, Comito G, Buricchi F, et al. Proliferation versus migration in platelet-derived growth factor signaling: the key role of endocytosis. J Biol Chem. 2008;283(29):19948-19956.

7. Dominici M, Le Blanc K, Mueller I, Slaper-Cortenbach I, Marini FC, Krause DS, Deans RJ, Keating A, Prockop DJ, Horwitz EM. Minimal criteria for defining multipotent mesenchymal stromal cells. The International Society for Cellular Therapy position statement. Cytotherapy. 2006; 8 (4); 315-317.

8. Du P, Kibbe WA, Lin SM. lumi: a pipeline for processing Illumina microarray. Bioinformatics 2008;24(13):1547-1548. 
9. Efımenko A.Yu., Dzhoyashvili N.A., Kalinina N.I., Kochegura T.N., Akchurin R.S., Tkachuk V.A., Parfyonova Ye.V. Adipose-derived stromal cells (ADSC) from aged patients with coronary artery disease keep MSC properties but exhibit age markers and have an impaired angiogenic potential. Stem Cells Translational Medicine. 2014; 3(1): 32-41.

10. Fafián-Labora JA and O'Loghlen A. Classical and Nonclassical Intercellular Communication in Senescence and Ageing. Trends in Cell Biology. 2020, in press.

11. Falcon S, Gentleman R. Using GOstats to test gene lists for GO term association. Bioinformatics 2007;23(2):257-258.

12. Gentleman RC, Carey VJ, Bates DM, Bolstad B, Dettling M, Dudoit S, Ellis B, Gautier L, Ge Y, Gentry J, Hornik K, Hothorn T, Huber W, lacus S, Irizarry R, Leisch F, Li C, Maechler M, Rossini AJ, Sawitzki G, Smith C, Smyth G, Tierney L, Yang JY, Zhang J. Bioconductor: open software development for computational biology and bioinformatics. Genome biology 2004;5(10):R80+.

13. Guillon J., Petit C., Moreau M. et al. Regulation of senescence escape by TSP1 and CD47 following chemotherapy treatment. Cell Death Dis 10, 199 (2019).

14. Heidaran MA, Pierce JH, Yu JC, et al. Role of alpha beta receptor heterodimer formation in beta plateletderived growth factor (PDGF) receptor activation by PDGF-AB. J Biol Chem. 1991;266(30):2023220237.

15. Kalinina N, Kharlampieva D, Loguinova M, et al. Characterization of secretomes provides evidence for adipose-derived mesenchymal stromal cells subtypes. Stem Cell Res Ther. 2015;6:221.

16. Klinkhammer BM, Floege J, Boor P. PDGF in organ fibrosis. Mol Aspects Med. 2018;62:44-62.

17. Kuhbier JW, Weyand B, Radtke C, Vogt PM, Kasper C, Reimers K. Isolation, characterization, differentiation, and application of adipose-derived stem cells. Adv Biochem Eng Biotechnol. 2010;123:55-105

18. Lin SM, Du P, Huber W, Kibbe WA. Model-based variance-stabilizing transformation for Illumina microarray data. Nucleic acids research 2008; 36(2): gkm1075+.

19. Macip, S., Igarashi, M., Fang, L., Chen, A., Pan, Z. Q., Lee, S. W., \& Aaronson, S. A. (2002). Inhibition of p21-mediated ROS accumulation can rescue p21-induced senescence. The EMBO journal, 21(9), 21802188.

20. Neri S. and Borzì R.M. Molecular Mechanisms Contributing to Mesenchymal Stromal Cell Aging. Biomolecules. 2020, 10, 340.

21. Pavón MA, Arroyo-Solera I, Céspedes MV, Casanova I, León X, Mangues R. UPA/uPAR and SERPINE1 in head and neck cancer: role in tumor resistance, metastasis, prognosis and therapy. Oncotarget. 2016;7(35):57351-57366.

22. Pierce, G.F., Mustoe, T.A., Altrock, B.W., Deuel, T.F. and Thomason, A. (1991), Role of platelet-derived growth factor in wound healing. J. Cell. Biochem., 45: 319-326.

23. Pittenger, M.F., Discher, D.E., Péault, B.M. et al. Mesenchymal stem cell perspective: cell biology to clinical progress. npj Regen Med. 2019, 4, 22. 
24. Sagaradze G, Basalova N, Kirpatovsky V, et al. A magic kick for regeneration: role of mesenchymal stromal cell secretome in spermatogonial stem cell niche recovery. Stem Cell Res Ther. 2019;10(1):342.

25. Song, G., Ouyang, G. and Bao, S. (2005), The activation of Akt/PKB signaling pathway and cell survival. Journal of Cellular and Molecular Medicine, 9: 59-71.

26. Vaughan DE, Rai R, Khan SS, Eren M, Ghosh AK. Plasminogen Activator Inhibitor-1 Is a Marker and a Mediator of Senescence. Arterioscler Thromb Vasc Biol. 2017;37(8):1446-1452.

27. Veevers-Lowe J, Ball SG, Shuttleworth A, Kielty CM. Mesenchymal stem cell migration is regulated by fibronectin through a5 $\beta 1$-integrin-mediated activation of PDGFR- $\beta$ and potentiation of growth factor signals. J Cell Sci. 2011;124(Pt 8):1288-1300.

28. Zuk PA, Zhu M, Mizuno H, Huang J, Futrell JW, Katz AJ, et al. Multilineage cells from human adipose tissue: implications for cell-based therapies. Tissue Eng. 2001;7:211-28

\section{Figures}
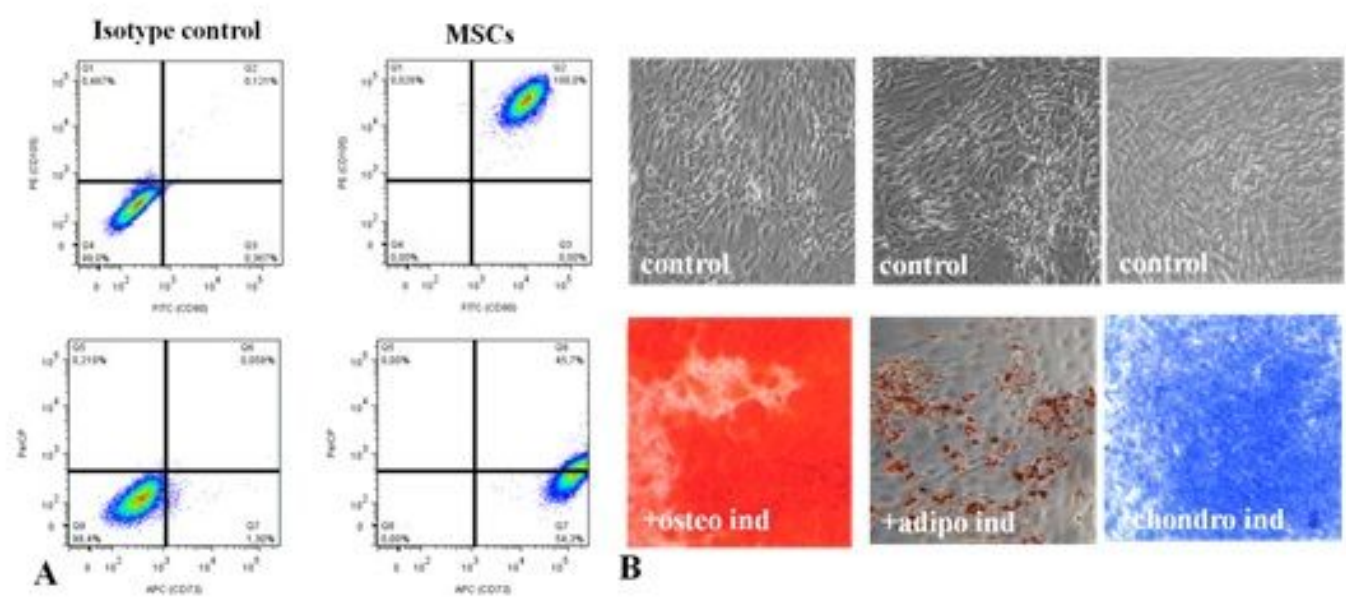

\section{Figure 1}

Human adipose-derived MSCs present characteristic features of multipotent mesenchymal stromal cells. A. Evaluation of MSC markers by flow cytometry. More than $99 \%$ of MSCs express CD73, CD90, CD105 on cell surface. B. MSCs differentiate into osteocytes (Alizarin Red S staining), adipocytes (Oil-Red-O staining) and chondrocytes (Toluidine Blue staining) under inductive conditions. Representative images, phase contrast microscopy. 


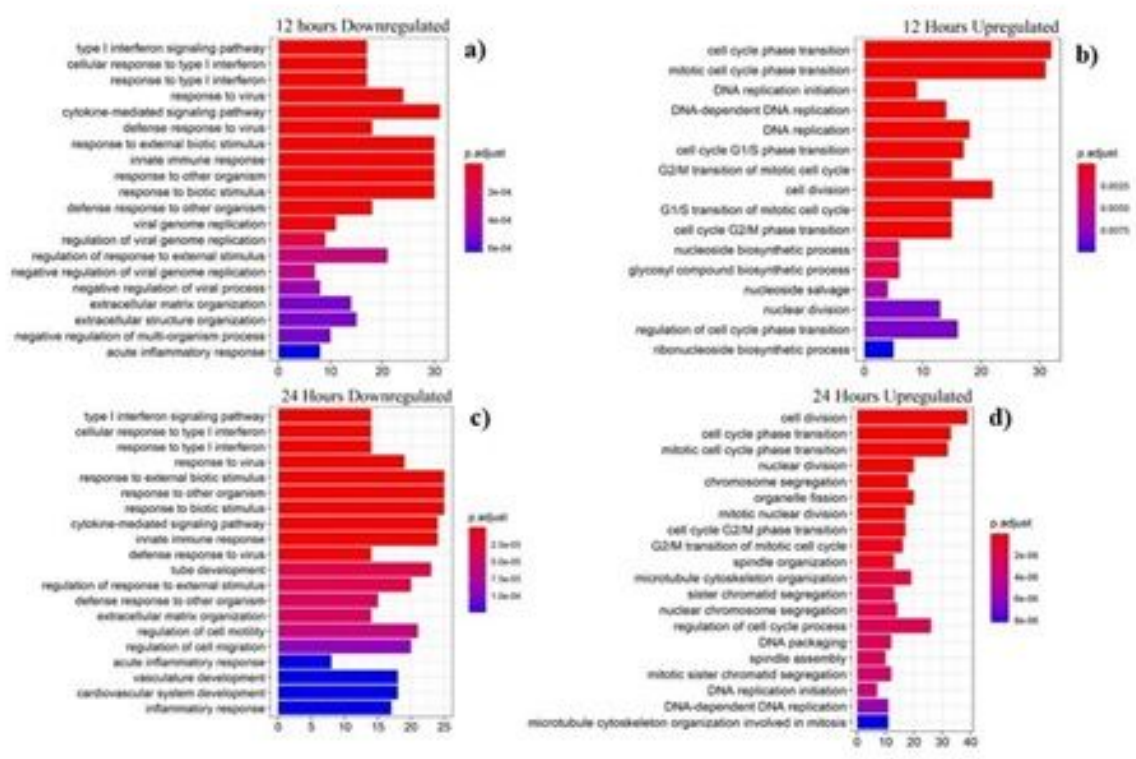

Figure 2

Biological processes associated with differentiatlly expressed genes in human adipose-derived MSCs after 12- and 24- hours of PDGF-BB treatment. a) 12 hours Downregulated GO BP barplot; b) 12 hours Upregulated GO BP barplot; c) 24 hours Downregulated GO BP barplot; d) 24 hours Upregulated GO BP barplot.

\section{Top GO BP terms for downregulated genes Top GO BP terms for upregulated genes}

12 type I interferon signaling pathway cellular response to type I interferon 10 response to type I interferon

8 response to virus

6 cytokine-mediated signaling pathway defense response to virus

4 response to external biotic stimulus

2 innate immune response

- response to other organism response to biotic stimulus defense response to other organism viral genome replication tube development regulation of response to extemal stimulus extracellular matrix organization regulation of cell motility regulation of cell migration regulation of viral genome replication acute inflammatory response inflammatory response

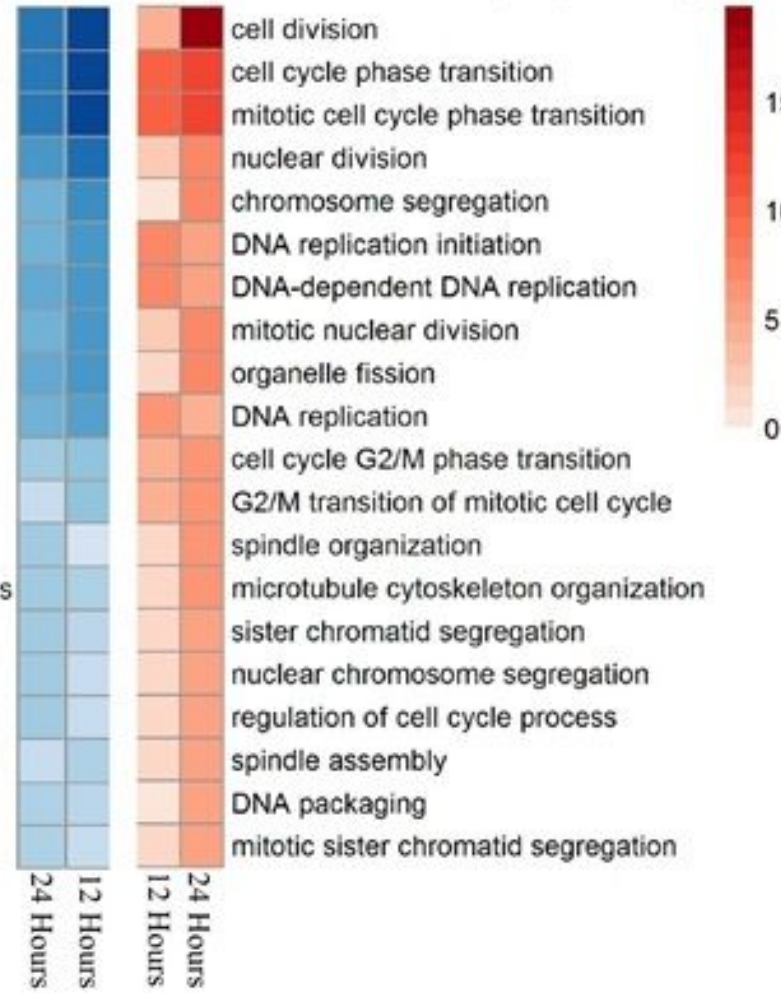


Figure 3

GO BP down/upregulated genes heatplot, 12- and 24- hours after PDGF-BB treatment of human adiposederived MSCs. The values represent -log10(p-value) for enrichment.
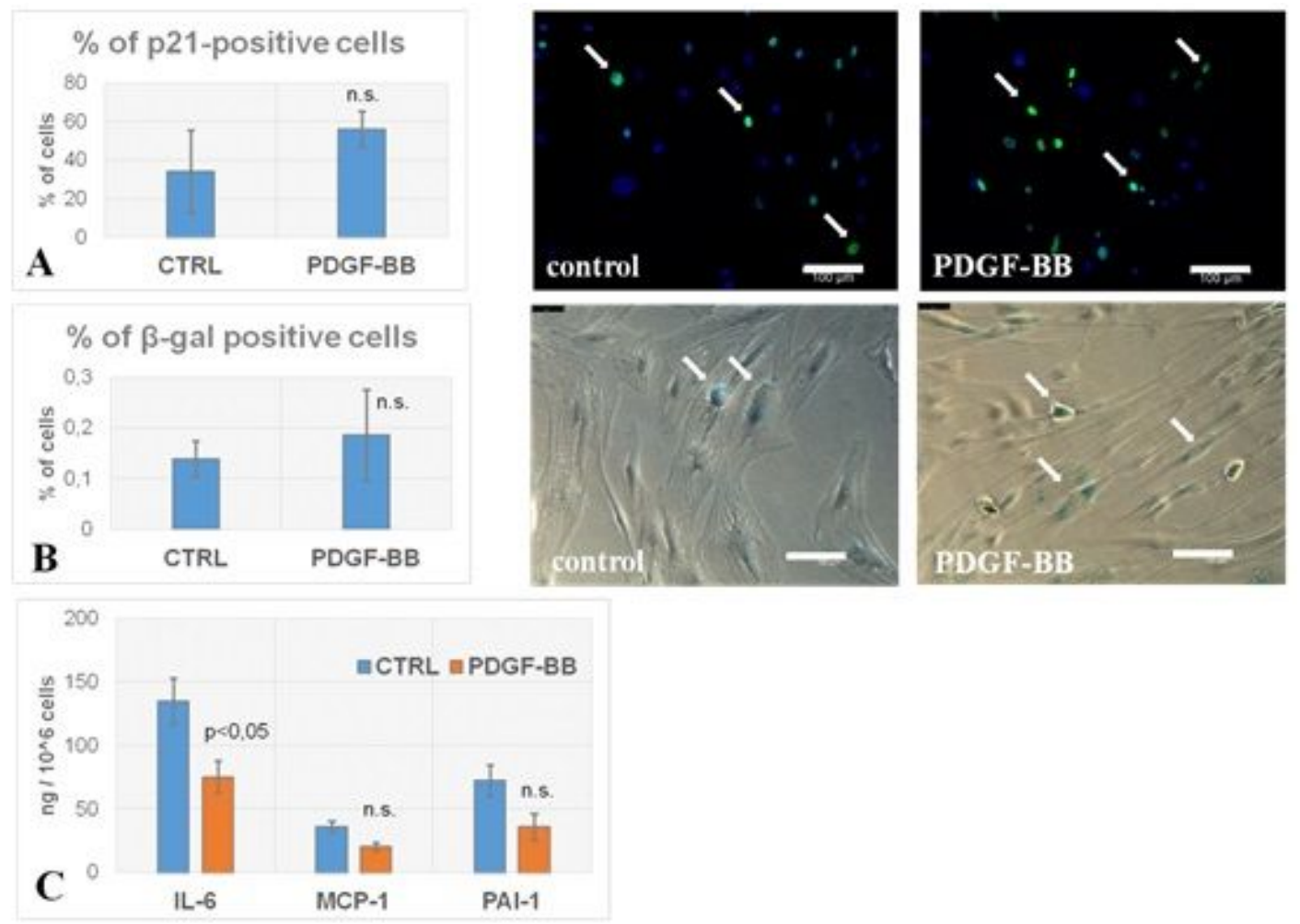

\section{Figure 4}

Biomarkers of senescence in PDFG-BB-treated MSCs. A. Immunocytochemical evaluation of p21Waf1/Cip1 in control (CTRL) MSCs and after incubation with PDGF-BB for 24 hours. Fluorescent microscopy, green staining - p21Waf1/Cip1, blue staining - nuclei stained with DAPI. The percentage of p21+cells was not changed significantly after PDGF-BB treatment. B. Evaluation of $\beta$-galactosidase activity (blue staining) in control (CTRL) MSCs and after incubation with PDGF-BB. Phase contrast microscopy. The percentage of $\beta-$ gal+ cells was not changed significantly after PDGF-BB treatment. C. MSC secretion of IL-6 decreased, while secretion of MCP-1 and PAI-1 was not changed significantly between control (CTRL) and after PDGF-BB treatment. Data are presented as mean value of three independent experiments, error bars - standard deviation SD (B) or standart error SE (A, C), n.s. - no significant difference. Scale bar $100 \mu \mathrm{m}$. 

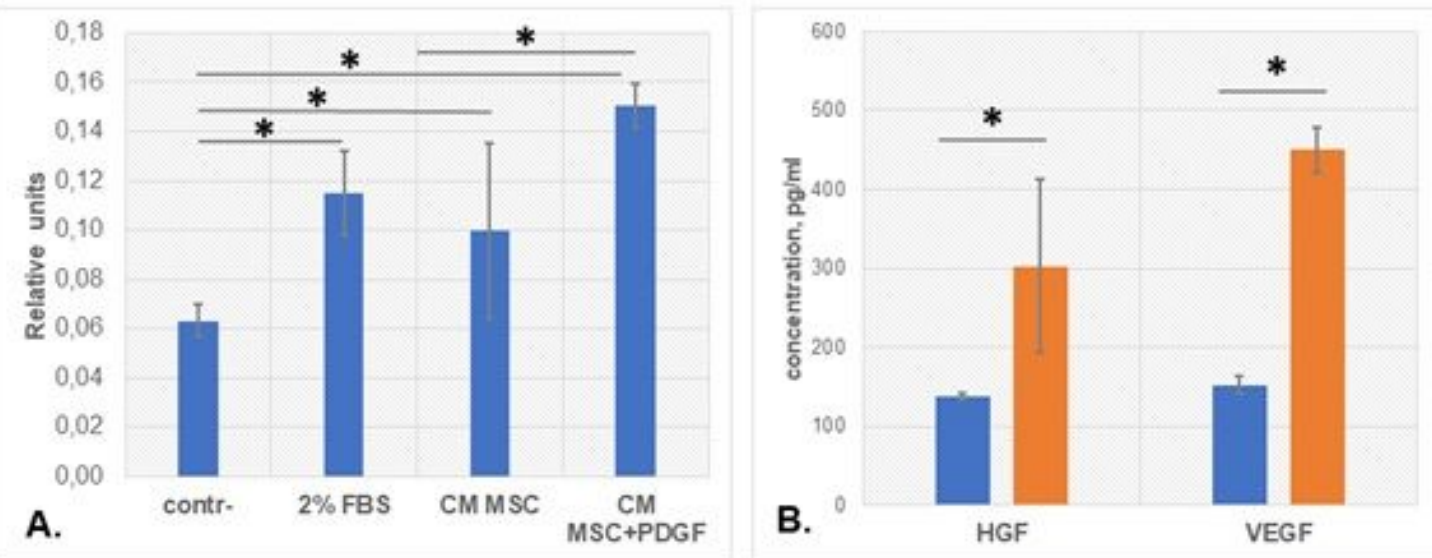

\section{Figure 5}

Analysis of MSC pro-angiogenic effects after PDGF-BB treatment. A. The slope (cell index, $\mathrm{Cl} /$ hour) of HUVEC migrating in CIM-plate of RTCA xCELLigence system towards medium conditioned by MSC (CM MSC) or medium conditioned by MSC after PDGF-BB treatment (CM MSC + PDGF). B. Concentration of growth factors HGF and VEGF in CM MSC (blue) and CM MSC + PDGF (orange). Data presented as mean value of three independent experiments, error bars - standard deviation $S \mathrm{D},{ }^{*} \mathrm{p}<0,05$. 